\title{
Keyword Index Volume 24 (2011)
}

\author{
abdominal drawing-in maneuver \\ abductor hallucis \\ anxiety \\ B200 isostation \\ back extensors \\ back pain \\ backpack \\ balneotherapy \\ blood pressure \\ bone mineral density \\ carpal tunnel syndrome \\ cervical epidural \\ cluneal nerve \\ cognitive-behavioural approach \\ complication \\ cross sectional area \\ curves \\ cycling \\ degeneration \\ depression \\ disability \\ disability evaluation \\ double pack \\ double-blind study \\ efficacy \\ electromyography \\ electrotherapy \\ epidural steroid injection \\ etiology \\ exercise therapy \\ exercises \\ fibromyalgia \\ fibromyalgia syndrome \\ front pack \\ gait analysis
}

233 healthy Nigerians $\quad 101$

healthy subjects $\quad 251$

herniated cervical disk $\quad 89$

hip osteoarthritis 201

hyaluronic acid $\quad 31$

hydrotherapy $\quad 57$

hypertension 111

intra-articular injection $\quad 31$

ketoprofen $\quad 39$

ketorolac 31

kinematic 201

knee 215

knee injury $\quad 49$

knee pain $\quad 77$

kyphosis 129

latent trigger point $\quad 251$

LBP 181

low back muscles 101

low back pain 83, 145, 195, 233

Lumbar range of motion $\quad 181$

lumbar region $\quad 241$

lumbar spine $\quad 155,195$

lumbosacral $\quad 67$

medial longitudinal arch $\quad 225$

morphotype $\quad 129$

MRI 23

multiple compression fractures $\quad 117$

$\begin{array}{ll}\text { muscle fat } & 23\end{array}$

muscle fatigue $\quad 17$

muscle strength 251

muscles 215

musculoskeletal disorders $\quad 161$

myofascial pain syndrome $\quad 257$

nerve entrapment 137

normative data 101 
Keyword Index Volume 24 (2011)

\author{
obesity \\ occupation \\ opioid \\ osteoarthritis \\ osteopoikilosis \\ pain \\ pain relief \\ patellar tendinopathy \\ percutaneous nucleotomy \\ peripheral nerve \\ pes planus \\ phonophoresis \\ physical therapy modalities \\ plain radiograph \\ post partum osteoporosis \\ posture \\ pulsed radiofrequency \\ pulsed radiofrequency technique \\ quadriceps muscle \\ quality of life \\ radicular irritation \\ rehabilitation \\ reliability \\ rheumatoid arthritis \\ roland-morris questionnaire \\ Rolland Morris \\ saphenous nerve \\ scale
}

111 scapular stabilization

173

49 sciatica 23

123 short-foot exercise 225

31,7

shoulder pain

189

Similarity Index (SI) $\quad 17$

sitting group exercise

181

$1,111,155$

Sørensen test

101

77

spine

209

4

spirometry

57

89

stability

209

static endurance

steroid

101

225

strengthening

123

57

Subacromial Impingement Syndrome

173

195

surface electromyography (EMG)

systematic review

173

transforaminal

67

189

215

95

transverse abdominal muscle

233

treatment

189

trigger point

137

ultrasound

39, 137, 233

89

$111,117,161$

validity

83,145

$83,145,195$

VAS

181

83

VAS score

181

walking

201

weight lifting

241

77

work hardening

161

145 work-related limitations 